\title{
On Some Classes of Double Difference Sequences of Interval Numbers
}

\author{
S. A. Mohiuddine, ${ }^{1}$ Kuldip Raj, ${ }^{2}$ and Abdullah Alotaibi ${ }^{1}$ \\ ${ }^{1}$ Department of Mathematics, Faculty of Science, King Abdulaziz University, P.O. Box 80203, Jeddah 21589, Saudi Arabia \\ ${ }^{2}$ School of Mathematics, Shri Mata Vaishno Devi University, Katra 182320, India
}

Correspondence should be addressed to S. A. Mohiuddine; mohiuddine@gmail.com

Received 9 May 2014; Accepted 27 June 2014; Published 24 July 2014

Academic Editor: Cristina Pignotti

Copyright ( 2014 S. A. Mohiuddine et al. This is an open access article distributed under the Creative Commons Attribution License, which permits unrestricted use, distribution, and reproduction in any medium, provided the original work is properly cited.

The aim of this paper is to introduce some interval valued double difference sequence spaces by means of Musielak-Orlicz function $\mathscr{M}=\left(M_{i j}\right)$. We also determine some topological properties and inclusion relations between these double difference sequence spaces.

\section{Introduction}

Interval arithmetic was first suggested by Dwyer [1] in 1951. Development of interval arithmetic as a formal system and evidence of its value as a computational device was provided by Moore [2] in 1959 and also by Moore and Yang [3] in 1962. Further works on interval numbers can be found in Dwyer [4] and Markov [5]. Furthermore, Moore and Yang [6] have developed applications of interval number sequences to differential equations. Chiao in [7] introduced sequences of interval numbers and defined usual convergence of sequences of interval number. Şengönül and Eryilmaz in [8] introduced and studied bounded and convergent sequence spaces of interval numbers and showed that these spaces are complete metric spaces. Recently, Esi in $[9,10]$ introduced and studied strongly almost $\lambda$-convergence and statistically almost $\lambda$ convergence of interval numbers and lacunary sequence spaces of interval numbers, respectively (also see [11-17]).

A set consisting of a closed interval of real numbers $x$ such that $a \leq x \leq b$ is called an interval number. A real interval can also be considered as a set. Thus we can investigate some properties of interval numbers, for instance, arithmetic properties or analysis properties. We denote the set of all real valued closed intervals by $\mathbb{R}$. Any elements of $\mathbb{R}$ are called closed interval and denoted by $\bar{x}$. That is, $\bar{x}=\{x \in \mathbb{R}: a \leq x \leq b\}$. An interval number $\bar{x}$ is a closed subset of real numbers [7]. Let $x_{l}$ and $x_{r}$ be first and last points of $\bar{x}$ interval number, respectively. For $\bar{x}_{1}, \bar{x}_{2} \in \mathbb{R}$, we have $\bar{x}_{1}=\bar{x}_{2} \Leftrightarrow x_{1_{l}}=x_{2_{l}}, x_{1_{r}}=x_{2_{r}}$. Consider $\bar{x}_{1}+\bar{x}_{2}=\left\{x \in \mathbb{R}: x_{1_{l}}+x_{2_{l}} \leq x \leq x_{1_{r}}+x_{2_{r}}\right\}$, and if $\alpha \geq 0$, then $\alpha \bar{x}=\left\{x \in \mathbb{R}: \alpha x_{1_{l}} \leq x \leq \alpha x_{1_{r}}\right\}$ and if $\alpha<0$, then $\alpha \bar{x}=\left\{x \in \mathbb{R}: \alpha x_{1_{r}} \leq x \leq \alpha x_{1_{l}}\right\}$,

$$
\begin{aligned}
\bar{x}_{1} \cdot \bar{x}_{2} \\
\quad=\left\{\begin{array}{r}
x \in \mathbb{R}: \min \left\{x_{1_{l}} \cdot x_{2_{l}}, x_{1_{l}} \cdot x_{2_{r}}, x_{1_{r}} \cdot x_{2_{l}}, x_{1_{r}} \cdot x_{2_{r}}\right\} \\
\leq x \leq \min \left\{x_{1_{l}} \cdot x_{2_{l}}, x_{1_{l}} \cdot x_{2_{r}}, x_{1_{r}} \cdot x_{2_{l}}, x_{1_{r}} \cdot x_{2_{r}}\right\}
\end{array}\right\} .
\end{aligned}
$$

In [2], Moore proved that the set of all interval numbers $\mathbb{R}$ is a complete metric space defined by $d\left(\bar{x}_{1}, \bar{x}_{2}\right)=\max \left\{\mid x_{1_{1}}-\right.$ $\left.x_{2_{l}}|,| x_{1_{r}} \cdot x_{2} \mid\right\}$. In the special cases $\bar{x}_{1}=[a, a]$ and $\bar{x}_{2}=[b, b]$, we obtain usual metric of $\mathbb{R}$. Let us define transformation $f$ : $\mathbb{N} \rightarrow \mathbb{R}$ by $k \rightarrow f(k)=\bar{x}, \bar{x}=\left(\bar{x}_{k}\right)$. Then $\bar{x}=\left(\bar{x}_{k}\right)$ is called sequence of interval numbers. The $\bar{x}_{k}$ is called $k$ th term of sequence $\bar{x}=\left(\bar{x}_{k}\right)$. We denote the set of all interval numbers with real terms as $w^{i}$. The algebraic properties of $w^{i}$ can be found in [7]. Now we give the basic definitions used in this paper.

Definition 1 (see [7]). A sequence $\bar{x}=\left(\bar{x}_{k}\right)$ of interval numbers is said to be convergent to the interval number $\bar{x}_{0}$ if for each $\epsilon>0$ there exists a positive integer $k_{0}$ such that 
$d\left(\bar{x}_{k}, \bar{x}_{0}\right)<\epsilon$ for all $k \geq k_{0}$ and we denote it by $\lim _{k} \bar{x}_{k}=\bar{x}_{0}$. Thus, $\lim _{k} \bar{x}_{k}=\bar{x}_{0} \Leftrightarrow \lim _{k} x_{k_{l}}=x_{0_{l}}$ and $\lim _{k} x_{k_{r}}=x_{0_{r}}$.

Definition 2. A transformation $f$ from $\mathbb{N} \times \mathbb{N}$ to $\mathbb{R}$ is defined by $i, j \rightarrow f(i, j)=\bar{x}, \bar{x}=\left(\bar{x}_{i j}\right)$. Then $\bar{x}=\left(\bar{x}_{i j}\right)$ is called sequence of double interval numbers. Then $\bar{x}_{i j}$ is called $i j^{\text {th }}$ term of sequence $\bar{x}=\left(\bar{x}_{i j}\right)$.

Definition 3. An interval valued double sequence $\bar{x}=\left(\bar{x}_{i j}\right)$ is said to be convergent in Pringsheim's sense or $P$-convergent to an interval number $\bar{x}_{0}$, if, for every $\epsilon>0$, there exists $N \epsilon$ $\mathbb{N}$ such that

$$
d\left(\bar{x}_{i j}, \bar{x}_{0}\right)<\epsilon \quad \forall i, j>N
$$

where $\mathbb{N}$ is the set of natural numbers, and we denote it also by $P-\lim \bar{x}_{i j}=\bar{x}_{0}$. The interval number $\bar{x}_{0}$ is called the Pringsheim limit of $\bar{x}=\left(\bar{x}_{i j}\right)$.

More exactly, we say that a double sequence $\bar{x}=\left(\bar{x}_{i j}\right)$ converges to a finite interval number $\bar{x}_{0}$ if $\bar{x}_{i j}$ tend to $\bar{x}_{0}$ as both $i$ and $j$ tend to $\infty$ independently of one another. We denote by $\bar{c}^{2}$ the set of all double convergent interval numbers of double interval numbers.

Definition 4. An interval valued double sequence $\bar{x}=\left(\bar{x}_{i j}\right)$ is bounded if there exists a positive number $M$ such that $d\left(\bar{x}_{i j}, \bar{x}_{0}\right) \leq M$ for all $i, j \in \mathbb{N}$. We will denote all bounded double interval number sequences by $\bar{l}_{\infty}^{2}$. It should be noted that, similar to the case of double sequences, $\bar{c}^{2}$ is not the subset of $\bar{l}_{\infty}^{2}$.

Definition 5. Let $A=\left(a_{m n i j}\right)$ denote a four-dimensional summability method that maps the complex double sequences $x$ into the double sequence $A x$ where the $m n$th term to $A x$ is as follows:

$$
(A x)_{m n}=\sum_{i, j=1,1}^{\infty, \infty} a_{m n i j} x_{i j}
$$

Such a transformation is said to be nonnegative if $a_{m n i j}$ is nonnegative for all $m, n, i$ and $j$.

The notion of difference sequence spaces was introduced by Kizmaz [18] who studied the difference sequence spaces $l_{\infty}(\Delta), c(\Delta)$, and $c_{0}(\Delta)$. The notion was further generalized by Et and Çolak [19] by introducing the spaces $l_{\infty}\left(\Delta^{n}\right), c\left(\Delta^{n}\right)$, and $c_{0}\left(\Delta^{n}\right)$. Let $w$ denote the set of all real and complex sequences and let $n$ be a nonnegative integer; then for $Z=c, c_{0}$, and $l_{\infty}$, we have sequence spaces

$$
Z\left(\Delta^{n}\right)=\left\{x=\left(x_{k}\right) \in w:\left(\Delta^{n} x_{k}\right) \in Z\right\},
$$

where $\Delta^{n} x=\left(\Delta^{n} x_{k}\right)=\left(\Delta^{n-1} x_{k}-\Delta^{n-1} x_{k+1}\right)$ and $\Delta^{0} x_{k}=x_{k}$ for all $k \in \mathbb{N}$, which is equivalent to the following binomial representation:

$$
\Delta^{n} x_{k}=\sum_{v=0}^{n}(-1)^{v}\left(\begin{array}{l}
n \\
v
\end{array}\right) x_{k+v}
$$

Taking $n=1$, we get the spaces studied by Et and Çolak [19]. For more details about sequence spaces see [20-32] and references therein. Quite recently, Et et al. [33] defined and studied the concept of statistical convergence of order $\alpha$ involving the notions of $\Delta$ and ideal $I$.

Definition 6. An Orlicz function $M:[0, \infty) \rightarrow[0, \infty)$ is a continuous, nondecreasing, and convex such that $M(0)=0$, $M(x)>0$ for $x>0$ and $M(x) \rightarrow \infty$ as $x \rightarrow \infty$. If convexity of Orlicz function is replaced by $M(x+y) \leq M(x)+M(y)$, then this function is called modulus function. Lindenstrauss and Tzafriri [34] used the idea of Orlicz function to define the following sequence space:

$\ell_{M}=\left\{x=\left(x_{k}\right) \in w: \sum_{k=1}^{\infty} M\left(\frac{\left|x_{k}\right|}{\rho}\right)<\infty\right.$, for some $\left.\rho>0\right\}$

which is known as an Orlicz sequence space. The space $\ell_{M}$ is a Banach space with the norm

$$
\|x\|=\inf \left\{\rho>0: \sum_{k=1}^{\infty} M\left(\frac{\left|x_{k}\right|}{\rho}\right) \leq 1\right\} .
$$

Also it was shown in [34] that every Orlicz sequence space $\ell_{M}$ contains a subspace isomorphic to $\ell_{p}(p \geq 1)$. An Orlicz function $M$ can always be represented in the following integral form:

$$
M(x)=\int_{0}^{x} \eta(t) d t
$$

where $\eta$ is known as the kernel of $M$ and is a right differentiable for $t \geq 0, \eta(0)=0, \eta(t)>0$, and $\eta$ is nondecreasing and $\eta(t) \rightarrow \infty$ as $t \rightarrow \infty$.

Definition 7. A sequence $\mathscr{M}=\left(M_{k}\right)$ of Orlicz functions is said to be Musielak-Orlicz function (see $[35,36])$. A sequence $\mathcal{N}=\left(N_{k}\right)$ is defined by

$$
N_{k}(v)=\sup \left\{|v| u-M_{k}(u): u \geq 0\right\}, \quad k=1,2, \ldots,
$$

and is called the complementary function of a MusielakOrlicz function $\mathscr{M}$. For a given Musielak-Orlicz function $\mathscr{M}$, the Musielak-Orlicz sequence space $t_{\mathscr{M}}$ and its subspace $h_{\mathscr{M}}$ are defined as follows:

$$
\begin{gathered}
t_{\mathscr{M}}=\left\{x \in w: I_{\mathscr{M}}(c x)<\infty \text { for some } c>0\right\}, \\
h_{\mathscr{M}}=\left\{x \in w: I_{\mathscr{M}}(c x)<\infty \forall c>0\right\},
\end{gathered}
$$

where $I_{\mathscr{M}}$ is a convex modular defined by

$$
I_{\mathscr{M}}(x)=\sum_{k=1}^{\infty} M_{k}\left(x_{k}\right), \quad x=\left(x_{k}\right) \in t_{\mathscr{M}} .
$$

We consider $t_{\mathscr{M}}$ equipped with the Luxemburg norm

$$
\|x\|=\inf \left\{k>0: I_{\mathscr{M}}\left(\frac{x}{k}\right) \leq 1\right\}
$$


or equipped with the Orlicz norm

$$
\|x\|^{0}=\inf \left\{\frac{1}{k}\left(1+I_{\mathscr{M}}(k x)\right): k>0\right\} .
$$

A Musielak-Orlicz function $\mathscr{M}=\left(M_{k}\right)$ is said to satisfy $\Delta_{2^{-}}$ condition if there exist constants $a, K>0$ and a sequence $c=$ $\left(c_{k}\right)_{k=1}^{\infty} \in l_{+}^{1}$ (the positive cone of $l^{1}$ ) such that the inequality

$$
M_{k}(2 u) \leq K M_{k}(u)+c_{k}
$$

holds for all $k \in \mathbb{N}$ and $u \in \mathbb{R}^{+}$, whenever $M_{k}(u) \leq a$.

Definition 8 . Let $X$ be a linear metric space. A function $p$ : $X \rightarrow \mathbb{R}$ is called paranorm, if

(1) $p(x) \geq 0$ for all $x \in X$;

(2) $p(-x)=p(x)$ for all $x \in X$;

(3) $p(x+y) \leq p(x)+p(y)$ for all $x, y \in X$;

(4) $\left(\lambda_{n}\right)$ is a sequence of scalars with $\lambda_{n} \rightarrow \lambda$ as $n \rightarrow \infty$ and $\left(x_{n}\right)$ is a sequence of vectors with $p\left(x_{n}-x\right) \rightarrow 0$ as $n \rightarrow \infty$, then $p\left(\lambda_{n} x_{n}-\lambda x\right) \rightarrow 0$ as $n \rightarrow \infty$.

A paranorm $p$ for which $p(x)=0$ implies $x=0$ is called total paranorm and the pair $(X, p)$ is called a total paranormed space. It is well known that the metric of any linear metric space is given by some total paranorm.

Let $\mathscr{M}=\left(M_{i j}\right)$ be a Musielak-Orlicz function and let $A=\left(a_{m n i j}\right)$ be a nonnegative four-dimensional bounded regular matrix (see $[37,38]$ ). Let $p=\left(p_{i j}\right)$ be a bounded double sequence of positive real numbers and $u=\left(u_{i j}\right)$ be a double sequence of strictly positive real numbers. In the present paper we define the following new double sequence spaces for interval sequences:

$$
\begin{aligned}
{ }_{2} \bar{w}\left(\mathscr{M}, p, u, \Delta^{r}, A\right) & \left\{\bar{x}=\left(\bar{x}_{i j}\right): P-\lim _{m n} \frac{1}{m n}\right. \\
& \times \sum_{i, j=1,1}^{m, n} a_{m n i j}\left[M_{i j}\left(\frac{u_{i j} d\left(\Delta^{r} \bar{x}_{i j}, \bar{x}_{0}\right)}{\rho}\right)\right]^{p_{i j}}=0, \\
& \text { for some } \rho>0\},
\end{aligned}
$$

$$
\begin{aligned}
& { }_{2} \bar{w}_{0}\left(\mathscr{M}, p, u, \Delta^{r}, A\right) \\
& =\left\{\bar{x}=\left(\bar{x}_{i j}\right): P-\lim _{m n} \frac{1}{m n}\right. \\
& \times \sum_{i, j=1,1}^{m, n} a_{m n i j}\left[M_{i j}\left(\frac{u_{i j} d\left(\Delta^{r} \bar{x}_{i j}, \overline{0}\right)}{\rho}\right)\right]^{p_{i j}}=0 \\
& \text { for some } \rho>0\} \text {, } \\
& { }_{2} \bar{w}_{\infty}\left(\mathscr{M}, p, u, \Delta^{r}, A\right) \\
& =\left\{\bar{x}=\left(\bar{x}_{i j}\right): \sup _{m n} \frac{1}{m n}\right. \\
& \times \sum_{i, j=1,1}^{m, n} a_{m n i j}\left[M_{i j}\left(\frac{u_{i j} d\left(\Delta^{r} \bar{x}_{i j}, \overline{0}\right)}{\rho}\right)\right]^{p_{i j}}<\infty, \\
& \text { for some } \rho>0\} \text {. }
\end{aligned}
$$

Remark 9. Let us consider a few special cases of the above sequence spaces.

(i) If $\mathscr{M}=M_{i j}(x)=x$ for all $i, j \in \mathbb{N}$, then we have

$$
\begin{gathered}
{ }_{2} \bar{w}\left(\mathscr{M}, p, u, \Delta^{r}, A\right)={ }_{2} \bar{w}\left(p, u, \Delta^{r}, A\right), \\
{ }_{2} \bar{w}_{0}\left(\mathscr{M}, p, u, \Delta^{r}, A\right)={ }_{2} \bar{w}_{0}\left(p, u, \Delta^{r}, A\right), \\
{ }_{2} \bar{w}_{\infty}\left(\mathscr{M}, p, u, \Delta^{r}, A\right)={ }_{2} \bar{w}_{\infty}\left(p, u, \Delta^{r}, A\right) .
\end{gathered}
$$

(ii) If $p=\left(p_{i j}\right)=1$, for all $i, j$, then we have

$$
\begin{gathered}
{ }_{2} \bar{w}\left(\mathscr{M}, p, u, \Delta^{r}, A\right)={ }_{2} \bar{w}\left(\mathscr{M}, u, \Delta^{r}, A\right), \\
{ }_{2} \bar{w}_{0}\left(\mathscr{M}, p, u, \Delta^{r}, A\right)={ }_{2} \bar{w}_{0}\left(\mathscr{M}, u, \Delta^{r}, A\right), \\
{ }_{2} \bar{w}_{\infty}\left(\mathscr{M}, p, u, \Delta^{r}, A\right)={ }_{2} \bar{w}_{\infty}\left(\mathscr{M}, u, \Delta^{r}, A\right) .
\end{gathered}
$$

(iii) If $u=\left(u_{i j}\right)=1$, for all $i, j$, then we have

$$
\begin{aligned}
{ }_{2} \bar{w}\left(\mathscr{M}, p, u, \Delta^{r}, A\right) & ={ }_{2} \bar{w}\left(\mathscr{M}, p, \Delta^{r}, A\right), \\
{ }_{2} \bar{w}_{0}\left(\mathscr{M}, p, u, \Delta^{r}, A\right) & ={ }_{2} \bar{w}_{0}\left(\mathscr{M}, p, \Delta^{r}, A\right), \\
{ }_{2} \bar{w}_{\infty}\left(\mathscr{M}, p, u, \Delta^{r}, A\right) & ={ }_{2} \bar{w}_{\infty}\left(\mathscr{M}, p, \Delta^{r}, A\right) .
\end{aligned}
$$

(iv) If $A=(C, 1,1)=1$, that is, the double Cesàro matrix, then the above classes of sequences reduce to the following sequence spaces:

$$
\begin{aligned}
{ }_{2} \bar{w}\left(\mathscr{M}, p, u, \Delta^{r}, A\right) & ={ }_{2} \bar{w}\left(\mathscr{M}, p, u, \Delta^{r}\right), \\
{ }_{2} \bar{w}_{0}\left(\mathscr{M}, p, u, \Delta^{r}, A\right) & ={ }_{2} \bar{w}_{0}\left(\mathscr{M}, p, u, \Delta^{r}\right), \\
{ }_{2} \bar{w}_{\infty}\left(\mathscr{M}, p, u, \Delta^{r}, A\right) & ={ }_{2} \bar{w}_{\infty}\left(\mathscr{M}, p, u, \Delta^{r}\right) .
\end{aligned}
$$


(v) Let $A=(C, 1,1)=1$ and $u_{i j}=1$ for all $i, j$. If, in addition, $\mathscr{M}(x)=M(x)$ and $r=0$, then the spaces ${ }_{2} \bar{w}\left(\mathscr{M}, p, u, \Delta^{r}, A\right), \quad{ }_{2} \bar{w}_{0}\left(\mathscr{M}, p, u, \Delta^{r}, A\right), \quad$ and ${ }_{2} \bar{w}_{\infty}\left(\mathscr{M}, p, u, \Delta^{r}, A\right)$ are reduced to ${ }_{2} \bar{w}(M, p)$, ${ }_{2} \bar{w}_{0}(M, p)$, and ${ }_{2} \bar{w}_{\infty}(M, p)$ which were introduced and studied by Esi and Hazarika [39].

The following inequality will be used throughout the paper. If $0 \leq p_{i j} \leq \sup p_{i j}=H, K=\max \left(1,2^{H-1}\right)$ then

$$
\left|a_{i j}+b_{i j}\right|^{p_{i j}} \leq K\left(\left|a_{i j}\right|^{p_{i j}}+\left|b_{i j}\right|^{p_{i j}}\right)
$$

for all $i, j$ and $a_{i j}, b_{i j} \in \mathbb{C}$. Also $|a|^{p_{i j}} \leq \max \left(1,|a|^{H}\right)$ for all $a \in \mathbb{C}$.

The main purpose of this paper is to introduce interval valued double difference sequence spaces ${ }_{2} \bar{w}\left(\mathscr{M}, p, u, \Delta^{r}, A\right)$, ${ }_{2} \bar{w}_{0}\left(\mathscr{M}, p, u, \Delta^{r}, A\right)$, and ${ }_{2} \bar{w}_{\infty}\left(\mathscr{M}, p, u, \Delta^{r}, A\right)$ and to study different properties of these spaces like linearity, paranorm, solidity, monotone, and so forth. Some inclusion relations between theses spaces are also established.

\section{Main Results}

Theorem 10. If $0<p_{i j}<q_{i j}$ for each $i$ and $j$, then we have ${ }_{2} \bar{w}_{\infty}\left(\mathscr{M}, p, u, \Delta^{r}, A\right) \subset{ }_{2} \bar{w}_{\infty}\left(\mathscr{M}, q, u, \Delta^{r}, A\right)$.

Proof. Let $\bar{x}=\left(\bar{x}_{i j}\right) \in{ }_{2} \bar{w}_{\infty}\left(\mathscr{M}, p, u, \Delta^{r}, A\right)$. Then there exists $\rho>0$ such that

$$
\sup _{m n} \frac{1}{m n} \sum_{i, j=1,1}^{m, n} a_{m n i j}\left[M_{i j}\left(\frac{u_{i j} d\left(\Delta^{r} \bar{x}_{i j}, \overline{0}\right)}{\rho}\right)\right]^{p_{i j}}<\infty .
$$

This implies that

$$
a_{m n i j}\left[M_{i j}\left(\frac{u_{i j} d\left(\Delta^{r} \bar{x}_{i j}, \overline{0}\right)}{\rho}\right)\right]^{p_{i j}}<1,
$$

for sufficiently large values of $i$ and $j$. Since $M_{i j}$ is nondecreasing, we get

$$
\begin{aligned}
& \sup _{m n} \frac{1}{m n} \sum_{i, j=1,1}^{m, n} a_{m n i j}\left[M_{i j}\left(\frac{u_{i j} d\left(\Delta^{r} \bar{x}_{i j}, \overline{0}\right)}{\rho}\right)\right]^{q_{i j}} \\
& \quad \leq \sup _{m n} \frac{1}{m n} \sum_{i, j=1,1}^{m, n} a_{m n i j}\left[M_{i j}\left(\frac{u_{i j} d\left(\Delta^{r} \bar{x}_{i j}, \overline{0}\right)}{\rho}\right)\right]^{p_{i j}}<\infty .
\end{aligned}
$$

Thus $\bar{x}=\left(\bar{x}_{i j}\right) \in{ }_{2} \bar{w}_{\infty}\left(\mathscr{M}, q, u, \Delta^{r}, A\right)$. This completes the proof.

Theorem 11. Suppose that $\mathscr{M}=\left(M_{i j}\right)$ is a Musielak-Orlicz function, $p=\left(p_{i j}\right)$ a bounded double sequence of positive real numbers, and $u=\left(u_{i j}\right)$ a double sequence of strictly positive real numbers. Then the following hold.

(i) If $0<\inf p_{i j}<p_{i j} \leq 1$, then ${ }_{2} \bar{w}_{\infty}\left(\mathscr{M}, p, u, \Delta^{r}, A\right) \subset$ ${ }_{2} \bar{w}_{\infty}\left(\mathscr{M}, u, \Delta^{r}, A\right)$. (ii) If $1 \leq p_{i j} \leq \sup p_{i j}<\infty$, then ${ }_{2} \bar{w}_{\infty}\left(\mathscr{M}, u, \Delta^{r}, A\right) \subset$ ${ }_{2} \bar{w}_{\infty}\left(\mathscr{M}, p, u, \Delta^{r}, A\right)$.

Proof. (i) Let $\bar{x}=\left(\bar{x}_{i j}\right) \in{ }_{2} \bar{w}_{\infty}\left(\mathscr{M}, p, u, \Delta^{r}, A\right)$. Since $0<$ inf $p_{i j} \leq 1$, we obtain the following:

$$
\begin{aligned}
& \sup _{m n} \frac{1}{m n} \sum_{i, j=1,1}^{m, n} a_{m n i j}\left[M_{i j}\left(\frac{u_{i j} d\left(\Delta^{r} \bar{x}_{i j}, \overline{0}\right)}{\rho}\right)\right] \\
& \quad \leq \sup _{m n} \frac{1}{m n} \sum_{i, j=1,1}^{m, n} a_{m n i j}\left[M_{i j}\left(\frac{u_{i j} d\left(\Delta^{r} \bar{x}_{i j}, \overline{0}\right)}{\rho}\right)\right]^{p_{i j}}<\infty,
\end{aligned}
$$

and hence $\bar{x}=\left(\bar{x}_{i j}\right) \in{ }_{2} \bar{w}_{\infty}\left(\mathscr{M}, u, \Delta^{r}, A\right)$.

(ii) Let $p_{i j} \geq 1$ for each $i$ and $j$ and $\sup p_{i j}<\infty$. Let $\bar{x}=\left(\bar{x}_{i j}\right) \in{ }_{2} \bar{w}_{\infty}\left(\mathscr{M}, u, \Delta^{r}, A\right)$. Then for each $0<\epsilon<1$ there exists a positive integer $N$ such that

$$
\begin{aligned}
& \sup _{m n} \frac{1}{m n} \sum_{i, j=1,1}^{m, n} a_{m n i j}\left[M_{i j}\left(\frac{u_{i j} d\left(\Delta^{r} \bar{x}_{i j}, \overline{0}\right)}{\rho}\right)\right] \\
& \leq \epsilon<1 \quad \forall n, m \geq N .
\end{aligned}
$$

This implies that

$$
\begin{aligned}
& \sup _{m n} \frac{1}{m n} \sum_{i, j=1,1}^{m, n} a_{m n i j}\left[M_{i j}\left(\frac{u_{i j} d\left(\Delta^{r} \bar{x}_{i j}, \overline{0}\right)}{\rho}\right)\right]^{p_{i j}} \\
& \quad \leq \sup _{m n} \frac{1}{m n} \sum_{i, j=1,1}^{m, n} a_{m n i j}\left[M_{i j}\left(\frac{u_{i j} d\left(\Delta^{r} \bar{x}_{i j}, \overline{0}\right)}{\rho}\right)\right]<\infty .
\end{aligned}
$$

Therefore, $\bar{x}=\left(\bar{x}_{i j}\right) \in{ }_{2} \bar{w}_{\infty}\left(\mathscr{M}, p, u, \Delta^{r}, A\right)$. This completes the proof.

Theorem 12. Let $0<p_{i j} \leq q_{i j}$ for all $i, j \in \mathbb{N}$ and $\left(q_{i j}\right)$ $\left.p_{i j}\right)$ be bounded. Then we have ${ }_{2} \bar{w}_{\infty}\left(\mathscr{M}, q, u, \Delta^{r}, A\right) \subset$ ${ }_{2} \bar{w}_{\infty}\left(\mathscr{M}, p, u, \Delta^{r}, A\right)$.

Proof. Let $\bar{x}=\left(\bar{x}_{i j}\right) \in{ }_{2} \bar{w}_{\infty}\left(\mathscr{M}, q, u, \Delta^{r}, A\right)$. Then

$$
\sup _{m n} \frac{1}{m n} \sum_{i, j=1,1}^{m, n} a_{m n i j}\left[M_{i j}\left(\frac{u_{i j} d\left(\Delta^{r} x_{i j}, \overline{0}\right)}{\rho}\right)\right]^{q_{i j}}<\infty,
$$

for some $\rho>0$.

Let $s_{i j}=\sup _{m n}(1 / m n) \sum_{i, j=1,1}^{m, n} a_{m n i j}\left[M_{i j}\left(u_{i j} d\left(\Delta^{r} \bar{x}_{i j}, \overline{0}\right) / \rho\right)\right]^{q_{i j}}$ and $\lambda_{i j}=p_{i j} / q_{i j}$. Since $p_{i j} \leq q_{i j}$, we have $0 \leq \lambda_{i j} \leq 1$. Take $0<\lambda<\lambda_{i j}$.

Define

$$
\begin{aligned}
& u_{i j}= \begin{cases}s_{i j} & \text { if } s_{i j} \geq 1 \\
0 & \text { if } s_{i j}<1,\end{cases} \\
& v_{i j}= \begin{cases}0 & \text { if } s_{i j} \geq 1 \\
s_{i j} & \text { if } s_{i j}<1,\end{cases}
\end{aligned}
$$


$s_{i j}=u_{i j}+v_{i j}, s_{i j}^{\lambda_{i j}}=u_{i j}^{\lambda_{i j}}+v_{i j}^{\lambda_{i j}}$. It follows that $u_{i j}^{\lambda_{i j}} \leq u_{i j} \leq s_{i j}$, $v_{i j}^{\lambda_{i j}} \leq v_{i j}^{\lambda}$. since $s_{i j}^{\lambda_{i j}}=u_{i j}^{\lambda_{i j}}+v_{i j}^{\lambda_{i j}}$, then $s_{i j}^{\lambda_{i j}} \leq s_{i j}+v_{i j}^{\lambda}$

$$
\begin{aligned}
& \sup _{m n} \frac{1}{m n} \sum_{i, j=1,1}^{m, n} a_{m n i j}\left[M_{i j}\left(\frac{u_{i j} d\left(\Delta^{r} \bar{x}_{i j}, \overline{0}\right)}{\rho}\right)^{q_{i j}}\right]^{\lambda_{i j}} \\
& \leq \sup _{m n} \frac{1}{m n} \sum_{i, j=1,1}^{m, n} a_{m n i j}\left[M_{i j}\left(\frac{u_{i j} d\left(\Delta^{r} \bar{x}_{i j}, \overline{0}\right)}{\rho}\right)\right]^{q_{i j}} \\
& \Longrightarrow \sup _{m n} \frac{1}{m n} \sum_{i, j=1,1}^{m, n} a_{m n i j}\left[M_{i j}\left(\frac{u_{i j} d\left(\Delta^{r} \bar{x}_{i j}, \overline{0}\right)}{\rho}\right)^{q_{i j}}\right]^{p_{i j} / q_{i j}} \\
& \leq \sup _{m n} \frac{1}{m n} \sum_{i, j=1,1}^{m, n} a_{m n i j}\left[M_{i j}\left(\frac{u_{i j} d\left(\Delta^{r} \bar{x}_{i j}, \overline{0}\right)}{\rho}\right)\right]^{q_{i j}} \\
& \Longrightarrow \sup _{m n} \frac{1}{m n} \sum_{i, j=1,1}^{m, n} a_{m n i j}\left[M_{i j}\left(\frac{u_{i j} d\left(\Delta^{r} \bar{x}_{i j}, \overline{0}\right)}{\rho}\right)\right]^{p_{i j}} \\
& \leq \sup _{m n} \frac{1}{m n} \sum_{i, j=1,1}^{m, n} a_{m n i j}\left[M_{i j}\left(\frac{u_{i j} d\left(\Delta^{r} \bar{x}_{i j}, \overline{0}\right)}{\rho}\right)\right]^{q_{i j}},
\end{aligned}
$$

but

$$
\begin{aligned}
& \sup _{m n} \frac{1}{m n} \sum_{i, j=1,1}^{m, n} a_{m n i j}\left[M_{i j}\left(\frac{u_{i j} d\left(\Delta^{r} \bar{x}_{i j}, \overline{0}\right)}{\rho}\right)\right]^{q_{i j}} \\
& <\infty \text { for some } \rho>0 .
\end{aligned}
$$

Therefore,

$$
\begin{aligned}
& \sup _{m n} \frac{1}{m n} \sum_{i, j=1,1}^{m, n} a_{m n i j}\left[M_{i j}\left(\frac{u_{i j} d\left(\Delta^{r} \bar{x}_{i j}, \overline{0}\right)}{\rho}\right)\right]^{p_{i j}} \\
& <\infty \text { for some } \rho>0 .
\end{aligned}
$$

Hence $\bar{x}=\left(\bar{x}_{i j}\right) \in{ }_{2} \bar{W}_{\infty}\left(\mathscr{M}, p, u, \Delta^{r}, A\right)$. Thus, we get ${ }_{2} \bar{W}_{\infty}\left(\mathscr{M}, q, u, \Delta^{r}, A\right) \subset{ }_{2} \bar{w}_{\infty}\left(\mathscr{M}, p, u, \Delta^{r}, A\right)$.

Theorem 13. Let $\mathscr{M}^{\prime}=\left(M_{i j}^{\prime}\right)$ and $\mathscr{M}^{\prime \prime}=\left(M_{i j}^{\prime \prime}\right)$ be two Musielak-Orlicz functions,

$$
\begin{gathered}
{ }_{2} \bar{w}_{\infty}\left(\mathscr{M}^{\prime}, p, u, \Delta^{r}, A\right) \cap{ }_{2} \bar{w}_{\infty}\left(\mathscr{M}^{\prime \prime}, p, u, \Delta^{r}, A\right) \\
\subset_{2} \bar{w}_{\infty}\left(\mathscr{M}^{\prime}+\mathscr{M}^{\prime \prime}, p, u, \Delta^{r}, A\right) .
\end{gathered}
$$

Proof. Let $\bar{x}=\left(\bar{x}_{i j}\right) \in{ }_{2} \bar{w}_{\infty}\left(\mathscr{M}^{\prime}, p, u, \Delta^{r}, A\right) \cap_{2} \bar{w}_{\infty}\left(\mathscr{M}^{\prime \prime}, p, u\right.$, $\left.\Delta^{r}, A\right)$. Then

$$
\begin{array}{r}
\sup _{m n} \frac{1}{m n} \sum_{i, j=1,1}^{m, n} a_{m n i j}\left[M_{i j}^{\prime}\left(\frac{u_{i j} d\left(\Delta^{r} \bar{x}_{i j}, \overline{0}\right)}{\rho_{1}}\right)\right]^{p_{i j}}<\infty, \\
\text { for some } \rho_{1}>0, \\
\sup _{m n} \frac{1}{m n} \sum_{i, j=1,1}^{m, n} a_{m n i j}\left[M_{i j}^{\prime \prime}\left(\frac{u_{i j} d\left(\Delta^{r} \bar{x}_{i j}, \overline{0}\right)}{\rho_{2}}\right)\right]^{p_{i j}}<\infty, \\
\text { for some } \rho_{2}>0 .
\end{array}
$$

Let $\rho=\max \left\{\rho_{1}, \rho_{2}\right\}$. The result follows from the inequality

$$
\begin{aligned}
\sup _{m n} & \frac{1}{m n} \sum_{i, j=1,1}^{m, n} a_{m n i j}\left[\left(M_{i j}^{\prime}+M_{i j}^{\prime \prime}\right)\left(\frac{u_{i j} d\left(\Delta^{r} \bar{x}_{i j}, \overline{0}\right)}{\rho}\right)\right]^{p_{i j}} \\
= & \sup _{m n} \frac{1}{m n} \sum_{i, j=1,1}^{m, n} a_{m n i j}\left[M_{i j}^{\prime}\left(\frac{u_{i j} d\left(\Delta^{r} \bar{x}_{i j}, \overline{0}\right)}{\rho_{1}}\right)\right]^{p_{i j}} \\
& +\sup _{m n} \frac{1}{m n} \sum_{i, j=1,1}^{m, n} a_{m n i j}\left[M_{i j}^{\prime \prime}\left(\frac{u_{i j} d\left(\Delta^{r} \bar{x}_{i j}, \overline{0}\right)}{\rho_{2}}\right)\right]^{p_{i j}} \\
\leq & K \sup _{m n} \frac{1}{m n} \sum_{i, j=1,1}^{m, n} a_{m n i j}\left[M_{i j}^{\prime}\left(\frac{u_{i j} d\left(\Delta^{r} \bar{x}_{i j}, \overline{0}\right)}{\rho_{1}}\right)\right]^{p_{i j}} \\
+ & K \sup _{m n} \frac{1}{m n} \sum_{i, j=1,1}^{m, n} a_{m n i j}\left[M_{i j}^{\prime \prime}\left(\frac{u_{i j} d\left(\Delta^{r} \bar{x}_{i j}, \overline{0}\right)}{\rho_{2}}\right)\right]^{p_{i j}} \\
<\infty . &
\end{aligned}
$$

Thus, $\sup _{m n}(1 / m n) \sum_{i, j=1,1}^{m, n} a_{m n i j}\left[\left(M_{i j}^{\prime}+M_{i j}^{\prime \prime}\right)\left(u_{i j} d\left(\Delta^{r} \bar{y}_{i j}, \overline{0}\right) /\right.\right.$ $\rho)]^{p_{i j}}<\infty$. Therefore, $\bar{x}=\left(\bar{x}_{i j}\right) \in{ }_{2} \bar{w}_{\infty}\left(\mathscr{M}^{\prime}+\mathscr{M}^{\prime \prime}, p\right.$, $\left.u, \Delta^{r}, A\right)$.

Theorem 14. Let $M=\left(M_{i j}\right)$ be a Musielak-Orlicz function and let $A=\left(a_{n m i j}\right)$ be a nonnegative four-dimensional regular summability method. Suppose that $\beta=\lim _{t \rightarrow \infty}\left(M_{i j}(t) / t\right)<$ $\infty$. Then ${ }_{2} \bar{w}\left(p, u, \Delta^{r}, A\right)={ }_{2} \bar{w}\left(\mathscr{M}, p, u, \Delta^{r}, A\right)$.

Proof. In order to prove that ${ }_{2} \bar{W}\left(p, u, \Delta^{r}, A\right)={ }_{2} \bar{w}(\mathscr{M}, p$, $\left.u, \Delta^{r}, A\right)$, it is sufficient to show that ${ }_{2} \bar{W}\left(\mathscr{M}, p, u, \Delta^{r}, A\right) \subset$ ${ }_{2} \bar{w}\left(p, u, \Delta^{r}, A\right)$. Now, let $\beta>0$. By definition of $\beta$, we have $M_{i j}(t) \geq \beta t$ for all $t \geq 0$. Since $\beta>0$, we have $t \leq(1 / \beta) M_{i j}(t)$ for all $t \geq 0$. Let $\bar{x}=\left(\bar{x}_{i j}\right) \in{ }_{2} \bar{w}\left(\mathscr{M}, p, u, \Delta^{r}, A\right)$. Thus, we have

$$
\begin{aligned}
& \sup _{m n} \frac{1}{m n} \sum_{i, j=1,1}^{m, n} a_{m n i j}\left[\left(\frac{u_{i j} d\left(\Delta^{r} \bar{x}_{i j}, \bar{x}_{0}\right)}{\rho}\right)\right]^{p_{i j}} \\
& \leq \frac{1}{\beta} \sup _{m n} \frac{1}{m n} \sum_{i, j=1,1}^{m, n} a_{m n i j}\left[M_{i j}\left(\frac{u_{i j} d\left(\Delta^{r} \bar{x}_{i j}, \bar{x}_{0}\right)}{\rho}\right)\right]^{p_{i j}}<\infty
\end{aligned}
$$


which implies that $\bar{x}=\left(\bar{x}_{i j}\right) \in{ }_{2} \bar{w}\left(p, u, \Delta^{r}, A\right)$. This completes the proof.

Theorem 15. Let $0<h=\inf p_{i j} \leq p_{i j} \leq \sup p_{i j}=H<\infty$. Then for a Musielak-Orlicz function $\mathscr{M}=\left(M_{i j}\right)$ which satisfies the $\Delta_{2}$-condition, we have ${ }_{2} \bar{w}\left(p, u, \Delta^{r}, A\right)={ }_{2} \bar{w}(\mathscr{M}, p, u$, $\left.\Delta^{r}, A\right)$.

Proof. Let $\bar{x}=\left(\bar{x}_{i j}\right) \in{ }_{2} \bar{w}\left(p, u, \Delta^{r}, A\right)$; that is,

$$
\frac{1}{m n} \sum_{i, j=1,1}^{m, n} a_{m n i j}\left[\left(\frac{u_{i j} d\left(\Delta^{r} \bar{x}_{i j}, \bar{x}_{0}\right)}{\rho}\right)\right]^{p_{i j}}=0,
$$

for some $\rho>0$.

Let $\epsilon>0$ and choose $\delta$ with $0<\delta<1$ such that $M_{i j}(t)<\epsilon$ for $0 \leq t \leq \delta$. Then

$$
\begin{aligned}
& \frac{1}{m n_{i, j=1,1}} \sum_{m n i j}^{m, n} a_{m n}\left[M_{i j}\left(\frac{u_{i j} d\left(\Delta^{r} \bar{x}_{i j}, \bar{x}_{0}\right)}{\rho}\right)\right]^{p_{i j}} \\
& =\frac{1}{m n} \sum_{\substack{i, j=1,1 \\
d\left(\Delta^{r} \bar{x}_{i j}, \bar{x}_{0}\right) \leq \delta}}^{m, n} a_{m n i j}\left[M_{i j}\left(\frac{u_{i j} d\left(\Delta^{r} \bar{x}_{i j}, \bar{x}_{0}\right)}{\rho}\right)\right]^{p_{i j}} \\
& +\frac{1}{m n} \sum_{\substack{i, j=1,1 \\
d\left(\Delta^{r} \bar{x}_{i j}, \bar{x}_{0}\right)>\delta}}^{m, n} a_{m n i j}\left[M_{i j}\left(\frac{u_{i j} d\left(\Delta^{r} \bar{x}_{i j}, \bar{x}_{0}\right)}{\rho}\right)\right]^{p_{i j}} \\
& =\sum_{1}+\sum_{2},
\end{aligned}
$$

where

$$
\begin{aligned}
\sum_{1} & =\frac{1}{m n} \sum_{\substack{i, j=1,1 \\
d\left(\Delta^{\top} \bar{x}_{i j}, \bar{x}_{0}\right) \leq \delta}}^{m, n} a_{m n i j}\left[M_{i j}\left(\frac{u_{i j} d\left(\Delta^{r} \bar{x}_{i j}, \bar{x}_{0}\right)}{\rho}\right)\right]^{p_{i j}} \\
& <\max \left(\epsilon, \epsilon^{H}\right)
\end{aligned}
$$

by using continuity of $\left(M_{i j}\right)$. For the second summation, we will make the following procedure. Thus we have

$$
\frac{d\left(\Delta^{r} x_{i j}, \bar{x}_{0}\right)}{\rho}<1+\frac{d\left(\Delta^{r} \bar{x}_{i j}, \bar{x}_{0}\right) / \rho}{\delta} .
$$

Since $\mathscr{M}=\left(M_{i j}\right)$ is nondecreasing and convex, so we have

$$
\begin{aligned}
a_{m n i j} & {\left[M_{i j}\left(\frac{u_{i j} d\left(\Delta^{r} \bar{x}_{i j}, \bar{x}_{0}\right)}{\rho}\right)\right] } \\
< & a_{m n i j}\left[M_{i j}\left\{1+\frac{u_{i j} d\left(\Delta^{r} \bar{x}_{i j}, \bar{x}_{0}\right) / \rho}{\delta}\right\}\right]
\end{aligned}
$$

$$
\begin{aligned}
\leq & \frac{1}{2} a_{m n i j}\left[\left(u_{i j}\right) M_{i j}(2)\right] \\
& +\frac{1}{2} a_{m n i j}\left[M_{i j}\left\{2 \frac{u_{i j} d\left(\Delta^{r} \bar{x}_{i j}, \bar{x}_{0}\right) / \rho}{\delta}\right\}\right] .
\end{aligned}
$$

Again, since $\mathscr{M}=\left(M_{i j}\right)$ satisfies the $\Delta_{2}$-condition, it follows that

$$
\begin{aligned}
& a_{m n i j}\left[M_{i j}\left(\frac{u_{i j} d\left(\Delta^{r} \bar{x}_{i j}, \bar{x}_{0}\right)}{\rho}\right)\right] \\
& \leq \frac{1}{2} K\left\{\frac{d\left(\Delta^{r} \bar{x}_{i j}, \bar{x}_{0}\right) / \rho}{\delta}\right\} a_{m n i j}\left[\left(u_{i j}\right) M_{i j}(2)\right] \\
&+\frac{1}{2} K\left\{\frac{d\left(\Delta^{r} \bar{x}_{i j}, \bar{x}_{0}\right) / \rho}{\delta}\right\} a_{m n i j}\left[\left(u_{i j}\right) M_{i j}(2)\right] \\
&= K\left\{\frac{d\left(\Delta^{r} \bar{x}_{i j}, \bar{x}_{0}\right) / \rho}{\delta}\right\} a_{m n i j}\left[\left(u_{i j}\right) M_{i j}(2)\right] .
\end{aligned}
$$

Thus, it follows that

$$
\begin{aligned}
\sum_{2}= & \max \left\{1,\left[\frac{K a_{m n i j}\left[\left(u_{i j}\right) M_{i j}(2)\right]}{\delta}\right]^{H}\right\} \\
& \times \frac{1}{m n} \sum_{i, j=1,1}^{m, n}\left[\frac{d\left(\Delta^{r} \bar{x}_{i j}, \bar{x}_{0}\right)}{\rho}\right]^{p_{i j}} .
\end{aligned}
$$

Taking the limit as $\epsilon \rightarrow 0$ and $m, n \rightarrow \infty$, it follows that $\bar{x}=\left(\bar{x}_{i j}\right) \in{ }_{2} \bar{w}\left(\mathscr{M}, p, u, \Delta^{r}, A\right)$.

Theorem 16. Suppose that $\mathscr{M}=\left(M_{i j}\right)$ is a Musielak-Orlicz function, $p=\left(p_{i j}\right)$ a bounded double sequence of positive real numbers, and $u=\left(u_{i j}\right)$ a double sequence of strictly positive real numbers. If $\sup _{i, j}\left(M_{i j}(x)\right)^{p_{i j}}<\infty$ for all fixed $x>0$, then

$$
{ }_{2} \bar{w}\left(\mathscr{M}, p, u, \Delta^{r}, A\right) \subset_{2} \bar{w}_{\infty}\left(\mathscr{M}, p, u, \Delta^{r}, A\right)
$$

Proof. Let $\bar{x}=\left(\bar{x}_{i j}\right) \in{ }_{2} \bar{w}\left(\mathscr{M}, p, u, \Delta^{r}, A\right)$. Then there exists a positive number $\rho_{1}>0$ such that

$$
\frac{1}{m n} \sum_{i, j=1,1}^{m, n} a_{m n i j}\left[M_{i j}\left(\frac{u_{i j} d\left(\Delta^{r} \bar{x}_{i j}, \bar{x}_{0}\right)}{\rho_{1}}\right)\right]^{p_{i j}}=0
$$


Define $\rho=2 \rho_{1}$. Since $\mathscr{M}=\left(M_{i j}\right)$ is nondecreasing and convex, for each $i, j$, so by using (20), we have

$$
\begin{gathered}
\sup _{m n} \frac{1}{m n} \sum_{i, j=1,1}^{m, n} a_{m n i j}\left[M_{i j}\left(\frac{u_{i j} d\left(\Delta^{r} \bar{x}_{i j}, \overline{0}\right)}{\rho}\right)\right]^{p_{i j}} \\
\leq \sup _{m n} \frac{1}{m n} \sum_{i, j=1,1}^{m, n} a_{m n i j}\left[M_{i j}\left(\frac{u_{i j} d\left(\Delta^{r} \bar{x}_{i j}, \bar{x}_{0}\right)+d\left(\bar{x}_{0}, \overline{0}\right)}{\rho}\right)\right]^{p_{i j}} \\
\leq K\left\{\sup _{m n} \frac{1}{m n} \sum_{i, j=1,1}^{m, n} a_{m n i j}\left[M_{i j}\left(\frac{u_{i j} d\left(\Delta^{r} \bar{x}_{i j}, \bar{x}_{0}\right)}{\rho_{1}}\right)\right]^{p_{i j}}\right. \\
\left.+\sup _{m n} \frac{1}{m n} \sum_{i, j=1,1}^{m, n} a_{m n i j}\left[M_{i j}\left(\frac{u_{i j} d\left(\Delta^{r} \bar{x}_{i j}, \overline{0}\right)}{\rho_{1}}\right)\right]^{p_{i j}}\right\}
\end{gathered}
$$

$<\infty$.

Thus $\bar{x}=\left(\bar{x}_{i j}\right) \in{ }_{2} \bar{w}_{\infty}\left(\mathscr{M}, p, u, \Delta^{r}, A\right)$. This completes the proof of the theorem.

Theorem 17. The double sequence space ${ }_{2} \bar{w}_{\infty}\left(\mathscr{M}, p, u, \Delta^{r}, A\right)$ is solid.

Proof. Suppose $\bar{x}=\left(\bar{x}_{i j}\right) \in{ }_{2} \bar{w}_{\infty}\left(\mathscr{M}, p, u, \Delta^{r}, A\right)$

$$
\sup _{m n} \frac{1}{m n} \sum_{i, j=1,1}^{m, n} a_{m n i j}\left[M_{i j}\left(\frac{u_{i j} d\left(\Delta^{r} \bar{x}_{i j}, \overline{0}\right)}{\rho}\right)\right]^{p_{i j}}<\infty
$$

for some $\rho>0$.

Let $\left(\alpha_{i j}\right)$ be a double sequence of scalars such that $\left|\alpha_{i j}\right| \leq 1$ for all $i, j \in \mathbb{N}$. Then we get

$$
\begin{aligned}
& \sup _{m n} \frac{1}{m n} \sum_{i, j=1,1}^{m, n} a_{m n i j}\left[M_{i j}\left(\frac{u_{i j} d\left(\Delta^{r} \alpha_{i j} \bar{x}_{i j}, \overline{0}\right)}{\rho}\right)\right]^{p_{i j}} \\
& \quad \leq \sup _{m n} \frac{1}{m n} \sum_{i, j=1,1}^{m, n} a_{m n i j}\left[M_{i j}\left(\frac{u_{i j} d\left(\Delta^{r} \bar{x}_{i j}, \overline{0}\right)}{\rho}\right)\right]^{p_{i j}} \\
& \quad<\infty .
\end{aligned}
$$

This completes the proof.

Theorem 18. The double sequence space ${ }_{2} \bar{w}_{\infty}\left(\mathscr{M}, p, u, \Delta^{r}, A\right)$ is monotone.

Proof. The proof is trivial so we omit it.

\section{Conflict of Interests}

The authors declare that there is no conflict of interests regarding the publication of this paper.

\section{Acknowledgment}

The authors gratefully acknowledge the financial support from King Abdulaziz University, Jeddah, Saudi Arabia.

\section{References}

[1] P. S. Dwyer, Linear Computation, Wiley, New York, NY, USA, 1951.

[2] R. E. Moore, Automatic Error Analysis in Digital Computation, LSMD-48421, Lockheed Missiles and Space Company, 1959.

[3] R. E. Moore and C. T. Yang, "Interval analysis I," Tech. Rep. LMSD-285875, Lockheed Missiles and Space Company, 1962.

[4] P. S. Dwyer, "Errors of matrix computation, simultaneous equations and eigenvalues, National Bureu of Standarts," Applied Mathematics Series, vol. 29, pp. 49-58, 1953.

[5] S. Markov, "Quasilinear spaces and their relation to vector spaces," Electronic Journal on Mathematics of Computation, vol. 2, no. 1, pp. 1-21, 2005.

[6] R. E. Moore and C. T. Yang, Theory of an Interval Algebra and Its Application to Numeric Analysis, RAAG Memories II, Gaukutsu Bunken Fukeyu-kai, Tokyo, Japan, 1958.

[7] K. P. Chiao, "Fundamental properties of interval vector maxnorm," Tamsui Oxford Journal of Mathematical Sciences, vol. 18, no. 2, pp. 219-233, 2002.

[8] M. Şengönül and A. Eryilmaz, "On the sequence spaces of interval numbers," Thai Journal of Mathematics, vol. 8, no. 3, pp. 503-510, 2010.

[9] A. Esi, "Strongly almost $\lambda$-convergence and statistically almost $\lambda$-convergence of interval numbers," Scientia Magna, vol. 7, no. 2, pp. 117-122, 2011.

[10] A. Esi, "Lacunary sequence spaces of interval numbers," Thai Journal of Mathematics, vol. 10, no. 2, pp. 445-451, 2012.

[11] A. Esi and B. Hazarika, "On interval valued generalized difference classes defined by Orlicz function," Turkish Journal of Analysis and Number Theory, vol. 1, no. 1, pp. 48-53, 2013.

[12] A. Esi and N. Braha, "On asymptotically $\lambda$-statistical equivalent sequences of interval numbers," Acta Scientiarum Technology, vol. 35, no. 3, pp. 515-520, 2013.

[13] A. Esi and A. Esi, "Asymptotically lacunary statistically equivalent sequences of interval numbers," International Journal of Applied Mathematics, vol. 1, no. 1, pp. 43-48, 2013.

[14] A. Esi, " $\lambda$-sequence spaces of interval numbers," Applied Mathematics \& Information Sciences, vol. 8, no. 3, pp. 1099-1102, 2014.

[15] A. Esi, "Double sequences of interval numbers defined by Orlicz functions," Acta et Commentationes Universitatis Tartuensis de Mathematica, vol. 17, no. 1, pp. 57-64, 2013.

[16] A. Esi and S. Yasemin, "Some spaces of sequences of interval numbers defined by a modulus function," Global Journal of Mathematical Analysis, vol. 2, no. 1, pp. 11-16, 2014.

[17] A. Esi, "Double lacunary sequence spaces of double sequence of interval numbers," Proyecciones. Journal of Mathematics, vol. 31, no. 3, pp. 299-308, 2012.

[18] H. Kizmaz, “On certain sequence spaces," Canadian Mathematical Bulletin, vol. 24, no. 2, pp. 169-176, 1981.

[19] M. Et and R. Çolak, "On some generalized difference sequence spaces," Soochow Journal of Mathematics, vol. 21, no. 4, pp. 377386, 1995.

[20] M. Et, "Generalized Cesàro difference sequence spaces of nonabsolute type involving lacunary sequences," Applied Mathematics and Computation, vol. 219, no. 17, pp. 9372-9376, 2013. 
[21] M. Et, "Spaces of Cesàro difference sequences of order $r$ defined by a modulus function in a locally convex space," Taiwanese Journal of Mathematics, vol. 10, no. 4, pp. 865-879, 2006.

[22] M. Et, Y. Altin, B. Choudhary, and B. C. Tripathy, "On some classes of sequences defined by sequences of Orlicz functions," Mathematical Inequalities \& Applications, vol. 9, no. 2, pp. 335342, 2006.

[23] V. Karakaya, E. Savas, and H. Polat, "Some paranormed Euler sequence spaces of difference sequences of order $m$," Mathematica Slovaca, vol. 63, no. 4, pp. 849-862, 2013.

[24] S. A. Mohiuddine and A. Alotaibi, "Some spaces of double sequences obtained through invariant mean and related concepts," Abstract and Applied Analysis, vol. 2013, Article ID 507950, 11 pages, 2013.

[25] S. A. Mohiuddine, K. Raj, and A. Alotaibi, "Some paranormed double difference sequence spaces for Orlicz functions and bounded-regular matrices," Abstract and Applied Analysis, vol. 2014, Article ID 419064, 10 pages, 2014.

[26] M. Mursaleen and S. A. Mohiuddine, "Some new double sequence spaces of invariant means," Glasnik Matematički, vol. 45, no. 1, pp. 139-153, 2010.

[27] M. Mursaleen, S. K. Sharma, and A. Kiliçman, "Sequence spaces defined by Musielak-Orlicz function over $n$-normed spaces," Abstract and Applied Analysis, vol. 2013, Article ID 364743, 10 pages, 2013.

[28] B. C. Tripathy, "Generalized difference paranormed statistically convergent sequences defined by Orlicz function in a locally convex space," Soochow Journal of Mathematics, vol. 30, no. 4, pp. 431-446, 2004.

[29] K. Raj and S. K. Sharma, "Some difference sequence spaces defined by Musielak-Orlicz functions," Mathematica Pannonica, vol. 24, pp. 33-43, 2013.

[30] K. Raj and S. K. Sharma, "Some multiplier double sequence spaces," Acta Mathematica Vietnamica, vol. 37, no. 3, pp. 391405, 2012.

[31] S. D. Parashar and B. Choudhary, "Sequence spaces defined by Orlicz functions," Indian Journal of Pure and Applied Mathematics, vol. 25, no. 4, pp. 419-428, 1994.

[32] S. Simons, "The sequence spaces $l\left(p_{v}\right)$ and $m\left(p_{v}\right)$," Proceedings of the London Mathematical Society, vol. 15, no. 3, pp. 422-436, 1965.

[33] M. Et, A. Alotaibi, and S. A. Mohiuddine, "On $\left(\triangle^{m}, I\right)$-statistical convergence of order $\alpha$," The Scientific World Journal, vol. 2014, Article ID 535419, 5 pages, 2014.

[34] J. Lindenstrauss and L. Tzafriri, "On Orlicz sequence spaces," Israel Journal of Mathematics, vol. 10, pp. 379-390, 1971.

[35] L. Maligranda, Orlicz Spaces and Interpolation, Seminars in Mathematics, vol. 5, Polish Academy of Science, Warsaw, Poland, 1989.

[36] J. Musielak, Orlicz Spaces and Modular Spaces, vol. 1034 of Lecture Notes in Mathematics, Springer, Berlin, Germany, 1983.

[37] H. J. Hamilton, “Transformations of multiple sequences," Duke Mathematical Journal, vol. 2, no. 1, pp. 29-60, 1936.

[38] G. M. Robison, "Divergent double sequences and series," Transactions of the American Mathematical Society, vol. 28, no. 1, pp. 50-73, 1926.

[39] A. Esi and B. Hazarika, "Some double sequence spaces of interval numbers defined by Orlicz functions," Journal of the Egyptian Mathematical Society, 2014. 


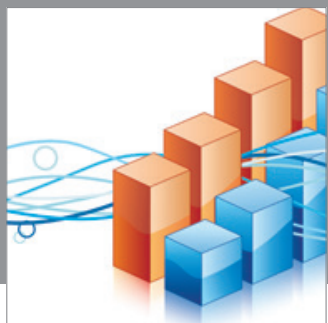

Advances in

Operations Research

mansans

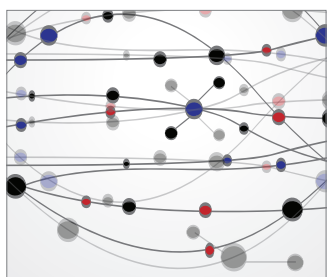

The Scientific World Journal
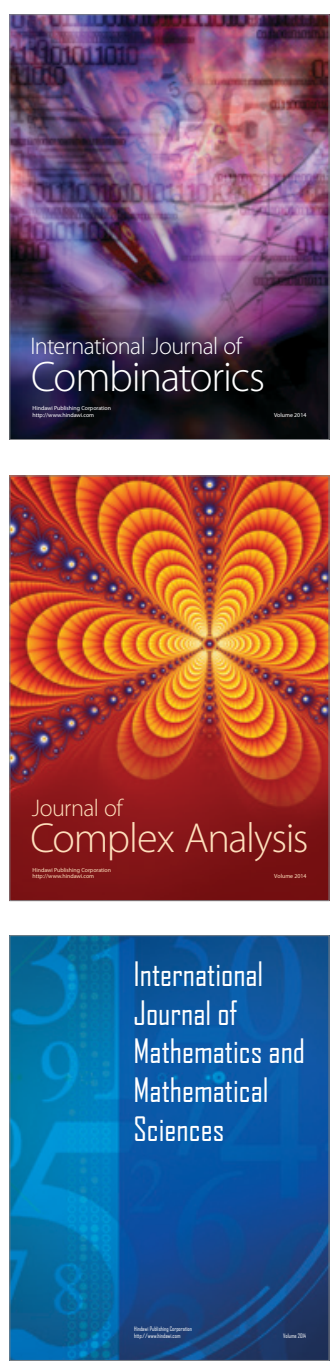
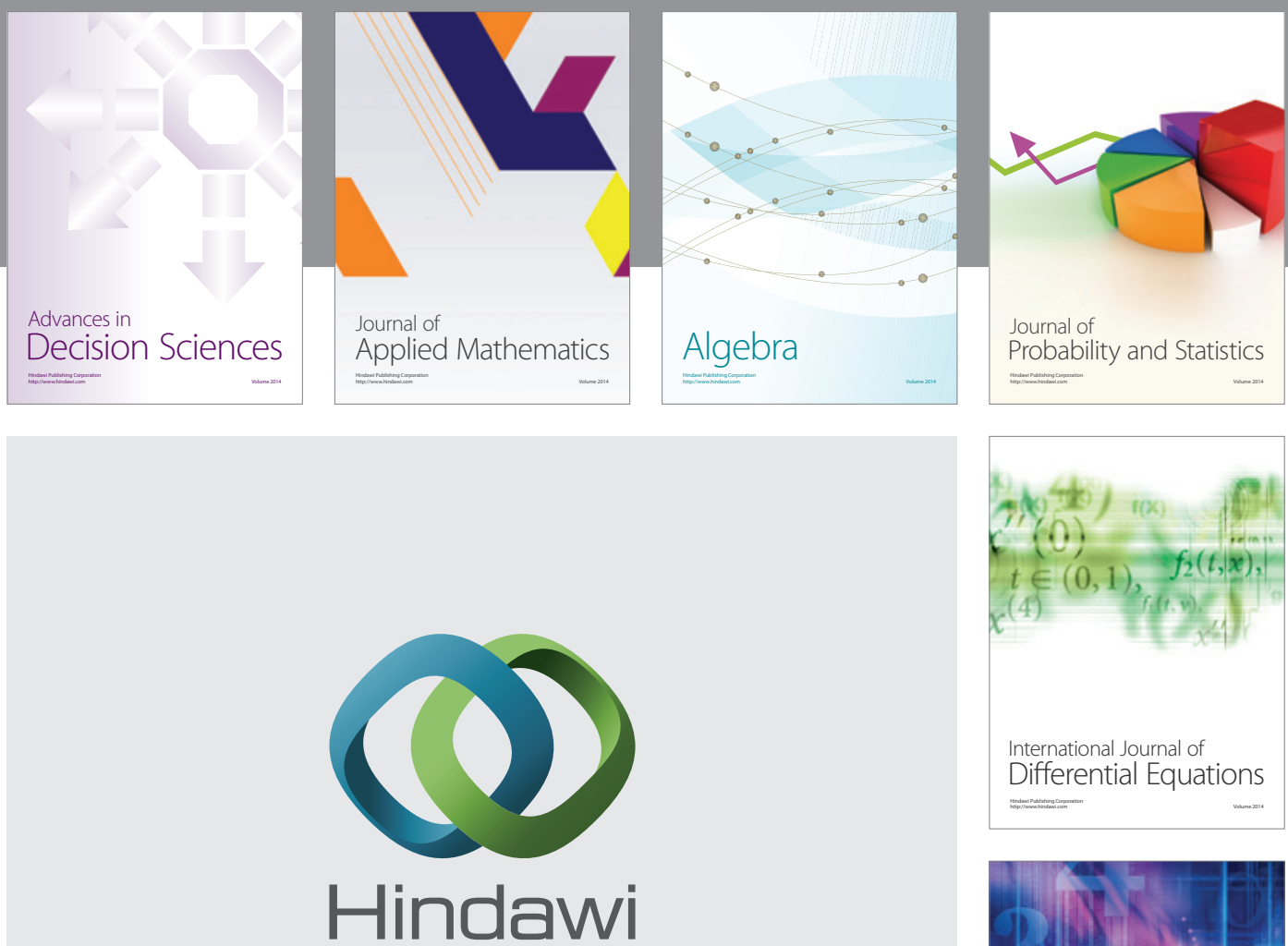

Submit your manuscripts at http://www.hindawi.com
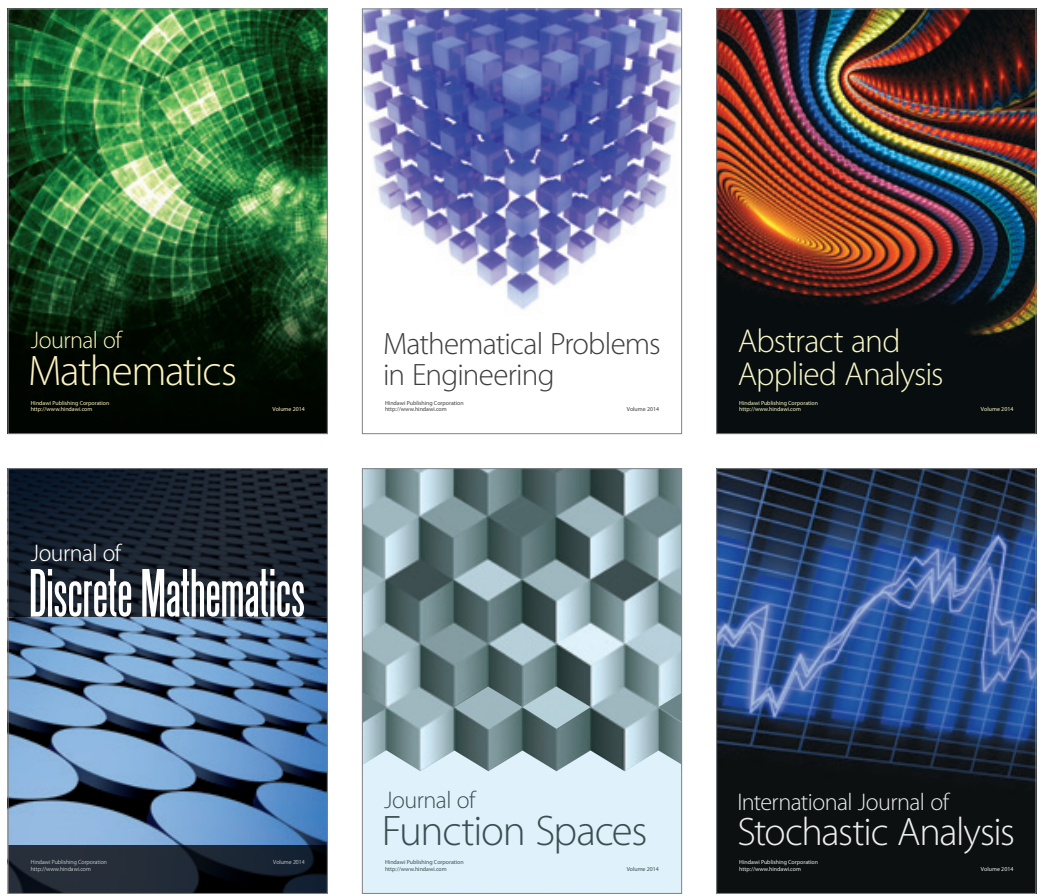

Journal of

Function Spaces

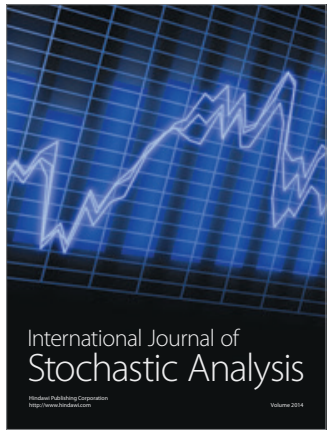

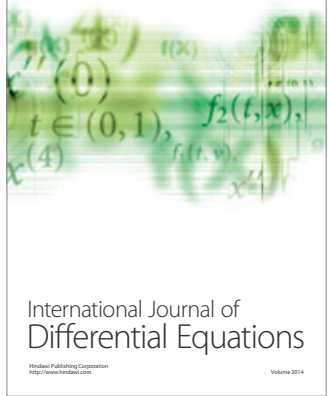
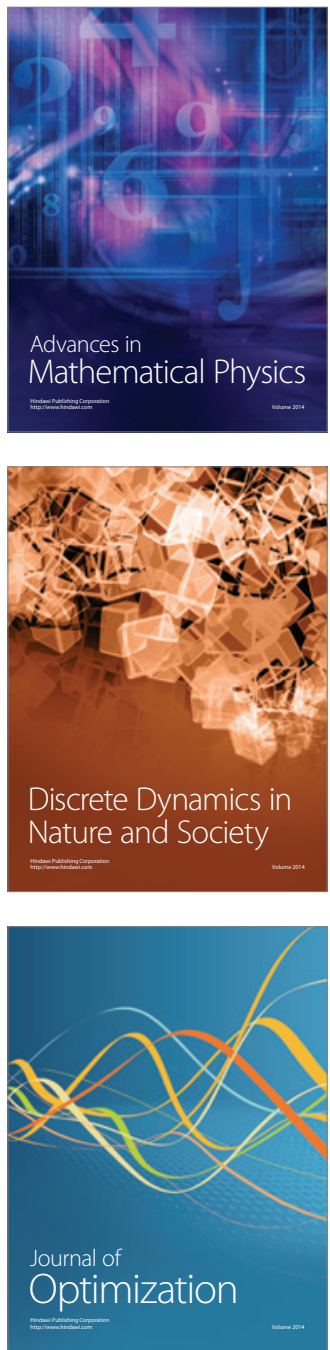